\title{
Dependence of the degree of paraxiality on field correlations
}

\author{
Omar El Gawhary ${ }^{1}$ and Sergio Severini ${ }^{2, *}$ \\ ${ }^{1}$ Optics Research Group, Department of Imaging Science and Technology, Delft University of Technology, \\ P.O. Box 5046, 2600 GA Delft, The Netherlands (O.ElGawhary@tudelft.nl) \\ ${ }^{2}$ Centro Interforze Studi per le Applicazioni Militari, Via della Bigattiera 10 (lato monte), \\ 56122 San Piero a Grado (Pi), Italy \\ *Corresponding author: s_severini@hotmail.com
}

Received May 20, 2008; accepted June 17, 2008;

posted July 9, 2008 (Doc. ID 96357); published August 8, 2008

A linearly polarized optical field can be obtained by filtering a stochastic field through an ideal linear polarizer. The produced field possesses a given degree of paraxiality that, as proved in the present Letter, can be affected by the correlations of the original stochastic field. An example with Gaussian beams is discussed in detail. (c) 2008 Optical Society of America

OCIS codes: $140.3300,350.5500$.

In recent times a good deal of study has been addressed toward the research of quantities providing a measure of the amount of paraxiality contained in a light beam [1-3]. Among them is the degree of paraxiality [4].

On supposing to deal with a field originating from a given input distribution on a starting source plane $(z=0)$, such a quantity, which we denote as $P$, is defined as the ratio between the time-averaged energy flow, across any transverse $(x, y)$ plane, carried by the nonparaxially propagated field and the corresponding paraxially propagated one. If $\mathbf{S}$ indicates the Poynting vector of a field under study, the said definition arises, in the case of monochromatic fields, from the condition $\nabla \cdot \mathbf{S}=0$; that implies the existence of particular invariants for the Helmholtz wave equation as well as its Fresnel approximation, called the paraxial wave equation. Interestingly, the calculation of this quantity can be performed only by knowing the plane-wave spectrum of the input-field distribution, owing to a useful application of Parseval's theorem. This means that it is not necessary to solve the paraxial or nonparaxial propagation problem to evaluate it. In addition, from the general theory we know that $P=0$ for a completely nonparaxial field, while $P=1$ for a fully paraxial one. In all other cases $0<P<1$, and the field is said to be partially paraxial. The degree of paraxiality just defined permits an easy comparison among different kinds of fields and allows us to understand which of them, and by how much, is the most paraxial (or, obviously, the most nonparaxial). Importantly, it can also be calculated in the case of sharp-edged fields (like, for instance, those produced by rectangular, circular, or annular slits) for which, on the contrary, other types of quality parameters, introduced in the past, suffer from some limitations [5].

Although in its original derivation [4] the degree of paraxiality was introduced in the case of monochromatic fields, its definition can be readily extended also to quasi-monochromatic fields for which the time-averaged value (on a time interval described in the following) of $\nabla \cdot \mathbf{S}$ still vanishes. In particular, for a scalar quasi-monochromatic field, endowed with a mean frequency $\bar{\nu}, P$ becomes

$$
P=\frac{\int_{p^{2}+q^{2}<1 / \lambda^{2}}\left\langle\left|A^{(0)}(p, q, t)\right|^{2}\right\rangle \sqrt{1-\bar{\lambda}^{2}\left(p^{2}+q^{2}\right)} \mathrm{d} p \mathrm{~d} q}{\int_{\infty}\left\langle\left|A^{(0)}(p, q, t)\right|^{2}\right\rangle \mathrm{d} p \mathrm{~d} q} .
$$

Here $A^{(0)}(p, q, t)$ is the plane-wave spectrum of the input field distribution, on $z=0 ; p$ and $q$ are the spatial frequencies in the Fourier space, and $\bar{\lambda}=c / \bar{\nu}$ is the mean wavelength. The angle brackets denote a time average over the interval $T=1 / \bar{\nu}$.

In this Letter we are interested in studying this topic from a different point of view. Our aim is indeed the investigation of the influence of field correlations on the paraxial quality of an optical beam. In particular we are going to prove the existence of a connection between the correlations of orthogonal field components of a stochastic, quasi-monochromatic beam and the paraxiality of the optical field obtained by sending the aforesaid stochastic field through a linear polarizer.

More precisely, let us consider a stationary, ergodic field with the following structure:

$$
\begin{aligned}
\mathbf{E}(x, y, z, t)= & E_{x}(x, y, z, t) \exp (-i 2 \pi \bar{\nu} t) \mathbf{x}_{0} \\
& +E_{y}(x, y, z, t) \exp (-i 2 \pi \bar{\nu} t) \mathbf{y}_{0} .
\end{aligned}
$$

The vector field $\mathbf{E}(x, y, z, t)$ is supposed to be quasimonochromatic with mean frequency $\bar{\nu}$. This implies that $E_{x}$ and $E_{y}$ are slowly varying functions of the $t$ variable. It is known that the second-order stochastic properties of a field like that in Eq. (2), on a given point $(x, y, z)$, are usually described by means of the $2 \times 2$ local polarization matrix $J_{\alpha \beta}=\left\langle E_{\alpha} E_{\beta}^{\star}\right\rangle$, where $\alpha, \beta=x, y$ and the asterisk indicates complex conjugation [6-9]. Let us suppose that the light beam in Eq. (2) passes through a linear polarizer, with the transmission axis making an angle $\theta$ with the $x$ axis. If the plane of the polarizer coincides with the $z=0$ plane, the emerging field, on $z=0^{+}$say, can be written as $E_{\theta}(x, y, 0, t)=E_{x}(x, y, 0, t) \cos \theta+E_{y}(x, y, 0, t) \sin \theta$. Owing to the linearity of the Fourier transformation, a 
similar expression can be written for its plane-wave spectrum:

$$
A_{\theta}^{(0)}(p, q, t)=A_{x}^{(0)}(p, q, t) \cos \theta+A_{y}^{(0)}(p, q, t) \sin \theta,
$$

where $A_{x}^{(0)}$ and $A_{y}^{(0)}$ are the plane-wave spectra, on $z$ $=0^{+}$, of the two orthogonal field components, $E_{x}$ and $E_{y}$. The time-averaged square modulus of $A_{\theta}^{(0)}$ results:

$$
\begin{aligned}
\left\langle\left|A_{\theta}^{(0)}\right|^{2}\right\rangle= & \left\langle\left|A_{x}^{(0)}\right|^{2}\right\rangle \cos ^{2} \theta+\left\langle\left|A_{y}^{(0)}\right|^{2}\right\rangle \sin ^{2} \theta \\
& +2 \operatorname{Re}\left\langle A_{x}^{(0)}\left(A_{y}^{(0)}\right)^{\star}\right\rangle \cos \theta \sin \theta,
\end{aligned}
$$

where Re denotes the real part. Putting Eq. (4) into Eq. (1), one can evaluate the degree of paraxiality $P$ of the field emerging from the polarizer. In particular, from Eq. (4) it is clear that $\left\langle\left|A_{\theta}^{(0)}\right|^{2}\right\rangle$ also depends on the existing correlation between the spectra $A_{x}^{(0)}$ and $A_{y}^{(0)}$. Such a correlation, represented by the mixed term $\left\langle A_{x}^{(0)}\left(A_{y}^{(0)}\right)^{\star}\right\rangle \cos \theta \sin \theta$, appears both into the numerator then into the denominator of Eq. (1). For this reason, one can expect to observe some modification of the degree of paraxiality $P$, induced by the polarization state of the original stochastic field. However, it is necessary to point out how correlations between the plane-wave spectra appearing in Eq. (4) are linked to those existing between the field components $E_{x}$ and $E_{y}$.

Since we have to evaluate integral energy flows, we are interested not in local correlation properties but in the global (or integral) ones. It is easily seen that existing correlations between $E_{x}(x, y, 0, t)$ and $E_{y}(x, y, 0, t)$ (and their linear transformations) can be directly transferred to $A_{x}^{(0)}$ and $A_{y}^{(0)}$. In fact, by using Parseval's theorem, one obtains, on $z=0$, $\int_{\infty}\left\langle E_{\alpha} E_{\beta}^{\star}\right\rangle \mathrm{d} x \mathrm{~d} y=\int_{\infty}\left\langle A_{\alpha}^{(0)}\left(A_{\beta}^{(0)}\right)^{\star}\right\rangle \mathrm{d} p \mathrm{~d} q$, with $\alpha, \beta=x, y$. It is important to realize that, if an integral correlation exists between $E_{\alpha}$ and $E_{\beta}$ on $z=0$, then the same must hold for their respective spectra. On the contrary, if two field components are globally uncorrelated, then their angular spectra will be uncorrelated, too. Similar reasoning can also be applied to integrals containing products between functions that are linear transformations of the given field components. In light of that, from now on, we focus ourselves only on $A_{x}^{(0)}$ and $A_{y}^{(0)}$ angular spectra and their stochastic properties.

To give an example of application of the theory, let us consider the case of Gaussian beams. In particular we assume that the stochastic field, incident on the polarizer, has the form given in Eq. (2) with the following terms:

$$
E_{\alpha}\left(x, y, 0^{-}, t\right)=A \exp \left(-r^{2} / w_{0 \alpha}^{2}\right) f_{\alpha}(t),
$$

with $r^{2}=x^{2}+y^{2}, \alpha=x, y$, and $A$ a constant value. In addition, with no loss of generality, we will assume $w_{0 x}=w_{0}$ and $w_{0 y}=a w_{0 x}$, where $a$ is a positive quantity. All the random features of the field are contained in the functions of time $f_{x}(t)$ and $f_{y}(t)$. They are endowed with a zero mean and fulfill the relationships $\left\langle\left|f_{x}(t)\right|^{2}\right\rangle=\sigma_{x}^{2}, \quad\left\langle\left|f_{y}(t)\right|^{2}\right\rangle=\sigma_{y}^{2}$, and $\left\langle f_{x}(t)\left(f_{y}(t)\right)^{\star}\right\rangle=\sigma_{x} \sigma_{y} \rho$. Here $\sigma_{x}^{2}$ and $\sigma_{y}^{2}$ are positive constants, while $\rho$ $=|\rho| \exp (i \varphi)$ is the complex correlation coefficient, with $0 \leqslant|\rho| \leqslant 1$. It's easily seen that for a field like that in Eq. (5), the angular spectra $A_{x}^{(0)}$ and $A_{y}^{(0)}$ are correlated between them just like the components $E_{x}$ and $E_{y}$ are. In this case Eq. (1) can be easily evaluated and turns out to be

$$
\begin{gathered}
\frac{\sigma_{x}^{2} \cos ^{2} \theta I_{G}^{\left(w_{0 x}\right)}+a^{2} \sigma_{y}^{2} \sin ^{2} \theta I_{G}^{\left(a w_{0 x}\right)}}{\sigma_{x}^{2} \cos ^{2} \theta+a^{2} \sigma_{y}^{2} \sin ^{2} \theta+4 \operatorname{Re}\{\rho\} \sigma_{x} \sigma_{y} \sin \theta \cos \theta \frac{a^{2}}{1+a^{2}}} \\
+\frac{4 \operatorname{Re}\{\rho\} \sigma_{x} \sigma_{y} \sin \theta \cos \theta \frac{a^{2}}{1+a^{2}} I_{G}^{\left(\sqrt{\left(1+a^{2}\right) / 2} w_{0}\right)}}{\sigma_{x}^{2} \cos ^{2} \theta+a^{2} \sigma_{y}^{2} \sin ^{2} \theta+4 \operatorname{Re}\{\rho\} \sigma_{x} \sigma_{y} \sin \theta \cos \theta \frac{a^{2}}{1+a^{2}}},
\end{gathered}
$$

where the following definition was used:

$$
I_{G}^{(w)}=1-\left(\frac{\bar{\lambda}}{2 \sqrt{2 \pi} w}\right) \exp \left(-\frac{2 \pi^{2} w^{2}}{\bar{\lambda}^{2}}\right) \operatorname{erf} i\left(\frac{\sqrt{2} \pi w}{\bar{\lambda}}\right) .
$$

In Eq. (7) we have introduced the function erfi(s) $=\operatorname{erf}(i s) / i$, with $\operatorname{erf}(s)$ the usual error function of argument $s$. A detailed discussion of Eq. (6) will permit us to put into evidence the main result of this Letter.

First, let us consider the case of completely unpolarized light (natural light). In this case it holds $\sigma_{x}$ $=\sigma_{y}, \rho=0$, and $a=1$, and the degree of paraxiality given by Eq. (6) simply reduces to $P=I_{G}^{\left(w_{0}\right)}$. The same result can also be obtained for a generic partially polarized light (i.e., for $\rho \neq 1$ and $\sigma_{x} \neq \sigma_{y}$ ) when $a=1$. As we will show soon, in all other situations, i.e., for $a$ $\neq 1$, the behavior of $P$ depends on the existing correlations between the orthogonal components into the original field. In particular, we are going to consider two limiting situations: the case of completely uncorrelated field components $(\rho=0)$ and that of fully correlated ones $(|\rho|=1)$. In this latter case, i.e., for completely polarized light, we will consider two different situations, corresponding to the $\varphi=0$ and $\varphi=\pi$ values.

In Fig. 1 the behavior of $P$, as a function of the adimensional variable $w_{0} / \bar{\lambda}$, for $\rho=-1$ and $\rho=0$ (when $\theta=\pi / 4$ and $a=2$ ), is reported. In the same figure, for the reader's convenience, we also report the behavior of $P$ for natural light. Plots show that, in all cases, the degree of paraxiality tends to unity when $w_{0} / \bar{\lambda}$ $\gg 1$, as it must be for Gaussian fields within the Fresnel regime [4]. However, it is evident that, within the nonparaxial region (characterized by values $w_{0} / \bar{\lambda} \ll 1$ ), the degree of paraxiality is considerably affected by the correlations in the original field. To make this aspect clearer, we plot in Fig. 2 the behavior of the difference ( $\Delta P$, e.g.,) between the degrees of paraxiality when $|\rho|=1$ and $\rho=0$. In particular, observing Fig. 2 we can see that there exists an 


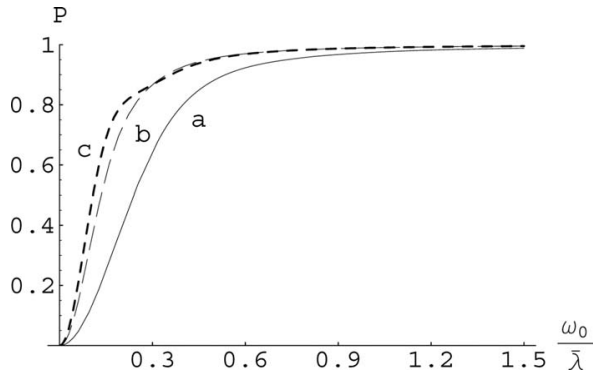

Fig. 1. Degree of paraxiality $P$ of a field produced by a stochastic optical beam passing through an ideal linear polarizer with transmission angle $\theta=\pi / 4$. We report (a) the behavior for natural light, (b) the plot for uncorrelated light ( $\rho=0, a=2$ ), and (c) the case of completely polarized light $(\rho=1, a=2$.). $\rho$ is the complex correlation coefficient for the components of the impinging field.

initial region, when $0<w_{0} / \bar{\lambda}<0.3$, where the light produced by a completely polarized field (with $\rho=-1$ ), is more paraxial than that originating from a partially polarized light made up of uncorrelated components. In this region, the maximum difference between the two degrees of polarization is about $13 \%$. More interestingly, the function $\Delta P$ changes its sign in the remaining region. In fact, when $0.3 \leqslant w_{0} / \bar{\lambda}$ $<\infty$, the amount of paraxiality produced by a partially polarized field, with uncorrelated components, is always greater than that due to the fully polarized field under consideration. This is a central, and somehow surprising, result of the present Letter. Actually,

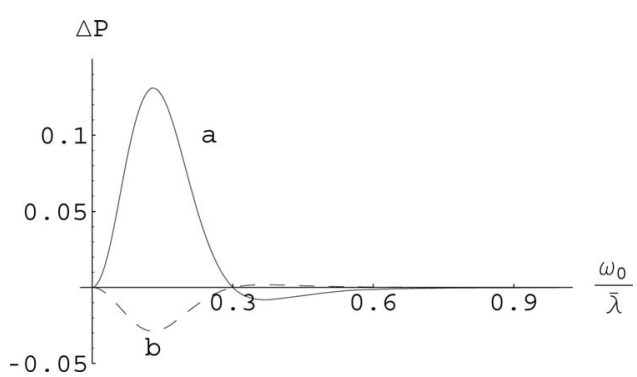

Fig. 2. Difference $\Delta P$ between the degree of paraxiality of fields produced by an original stochastic optical beam passing through an ideal linear polarizer with transmission angle $\theta=\pi / 4$. In (a) we report such a difference between a fully polarized original field $(\rho=-1)$ and a partially polarized one endowed with uncorrelated orthogonal field components $(\rho=0)$. In (b) the behavior of $\Delta P$ in the case of a fully polarized original field (but with $\rho=1$ ) and a partially polarized one endowed with uncorrelated orthogonal field components $(\rho=0)$ is shown. With reference to Eq. (6) we have set $a=2$. it simply means that, under certain conditions, some stochasticity can improve the paraxial quality of a light beam. In other words, one can barter degree of polarization with degree of paraxiality. This proves that field correlations, present in the original field, can actually affect the degree of paraxiality of the field emerging from the polarizer. As to the other case, when the fully polarized field has $\rho=1$ (e.g., $|\rho|$ $=1$ and $\varphi=0$ ), the degree of paraxiality associated with uncorrelated light is initially greater than that of fully polarized light, although the maximum of the difference $\Delta P$ is notably smaller than the previous case (see Fig. 2). Then, again in correspondence with $w_{0} / \bar{\lambda} \approx 0.3$, the situation reverses and the polarized light becomes the most paraxial. Owing to the difference found for the cases $|\rho|=1, \varphi=0$, and $|\rho|=1, \varphi=\pi$, we can conclude that the degree of paraxiality depends not only on the modulus of the complex correlation coefficient $\rho$ but also on its phase $\varphi$. For the example being considered here, the maximum difference between correlated and uncorrelated light is reached for $|\rho|=1$ and $\varphi=\pi$. All these results clearly show a connection between paraxiality and field correlations, which represents the aim of the present Letter.

In summary, the effect of field correlations on the degree of paraxiality has been studied in detail. In particular, we have proved how the degree of paraxiality of a light beam, filtered by a linear polarizer, can be affected by the polarization state of the incident field on the polarizer itself. We have discussed the general theory and we have given a valid example with quasi-monochromatic mutually orthogonal Gaussian fields. The obtained results have shown that the degree of paraxiality of the emerging optical field depends on the modulus of the complex correlation coefficient as well as on its phase.

\section{References}

1. P. Vaveliuk, B. Ruiz, and A. Lencina, Opt. Lett. 32, 927 (2007)

2. S. R. Seshadri, Appl. Opt. 45, 5335 (2006).

3. O. El Gawhary and S. Severini, Appl. Opt. 47, 3767 (2008).

4. O. El Gawhary and S. Severini, Opt. Lett. 33, 1360 (2008)

5. C. J. R. Sheppard, J. Opt. Soc. Am. A 18, 1579 (2001).

6. M. Born and E. Wolf, Principles of Optics, 7th ed. (Cambridge U. Press, 2001).

7. L. Mandel and E. Wolf, Optical Coherence and Quantum Optics (Cambridge U. Press, 1995).

8. E. Wolf, Nuovo Cimento 12, 884 (1954).

9. F. Gori, Opt. Lett. 4, 241 (1997). 